\title{
Expert System for Early Diagnosis of COVID - 19
}

\section{Sharana Dharshikgan Suresh Dass ${ }^{1}$, Fatemeh Meskaran ${ }^{1}$, Mitra Saeedi ${ }^{1}$}

\section{IJCRR}

Section: Healthcare

Sci. Journal Impact

Factor: 6.1 (2018)

ICV: 90.90 (2018)

\author{
'Asia Pacific University of Technology and Innovation.
}

\section{ABSTRACT}

COVID-19 is an illness caused by a novel coronavirus also known as severe acute respiratory syndrome coronavirus 2, which was first identified in the City of Wuhan, China. Since then, it has been declared as a global pandemic by the World Health Organization. The late diagnosis of COVID-19 patients makes the fast spreading of the virus across the globe. Purpose: Thus, the solution to slow down the spread of this virus would be an expert system that will be able to diagnose COVID - 19 patients and produce results instantly. This paper discusses how a knowledge-based expert system can help to diagnose or detect COVID 19 in the early stage itself and get the result immediately without any delay.

Key Words: Artificial intelligence, Expert system, Medical diagnosis, Hybrid intelligent systems, Knowledge acquisition, COVID-19

\section{INTRODUCTION}

The novel coronavirus disease (COVID-19) has created tremendous chaos around the world, affecting people's lives and causing a large number of deaths. Its first cases were detected in Wuhan, China in December 2019 and now it has been spread to almost every country. Governments of many countries have proposed policies to mitigate the impacts of the COVID-19 pandemic. Science and technology have contributed significantly to the implementations of these policies during this unprecedented and chaotic time.

The coronavirus (COVID-19) has made exceptional clutter around the world. The primary case was distinguished in Wuhan, China in December 2019 and it has been spread all around the world. Distinctive arrangements and rules are proposed concerning dealing with COVID-19 by governments. Science and innovation have supported. Early detection and evaluation of infected patients have been considered by most of the computer science researchers. The artificial intelligence (AI) and different related computational techniques play a considerable role for this purpose. In general, when detecting disease at the later stage is much complicated to treat compared to detecting the disease at an earlier stage. The same concept applies to detect COVID-19. Based on the Malaysian newspaper (The STAR, 2020) the main cause of death in Malaysia due to COVID-19 is because of late treatment. ${ }^{1}$ This also means that the patients were diagnosed with the virus very much later and not in the beginning stage. Also, late detection of the virus might put other people live at risk. This is because when the potential patient does not know if he or she is infected with the virus, they might carry on with their daily routine and spreading the virus to others.

Besides that, when there is a delay in the detection of viruses in older people and those with underlying medical problems like cardiovascular disease, diabetes, chronic respiratory disease, and cancer, they are more likely to develop serious illness. ${ }^{2}$ This also increases the workload of the front liners in the medical field as the treatments for these cases are much more complicated than the normal cases suffering from COVID-19 and require more and special attention and medical care. Furthermore, the reason why the current detection method is not so efficient is that multiple processes need to be carried out before an actual result can be obtained. Based on NPR Choice report in 2020, the first step that needs to be carried out is to take a sample from a patient's nose or throat, using a special swab. That swab goes into a tube and is sent to a lab. ${ }^{3}$ Small hospitals mostly send their samples to

\section{Corresponding Author:}

Sharana Dharshikgan Suresh Dass, Asia Pacific University of Technology and Innovation.

ISSN: 2231-2196 (Print)

Received: 22.08 .2020
ISSN: 0975-5241 (Online)

Revised: 20.09 .2020
Accepted: 05.11 .2020
Published: 24.11 .2020 
outside laboratories for processing but large hospitals usually have on-site molecular test labs, however, The transit time depends on how far the hospital is from the processing laboratory and usually takes around 1 day. Then, in the laboratory, a chemical test called polymerase chain reaction (PCR) is done. This is the test that determines if the sample is COVID-19 positive or negative. Besides that, the PCR usually takes 6 hours to get the result. The whole process of detecting the COVID-19 takes around 2 to 3 days which is still quite lengthy. By then, the infected patient could spread the virus to many others around him or her.

Thus, the technique that can potentially solve this issue is from the domain of Artificial Intelligence which is the Expert System. This paper focuses on the roles of AI technologies and especially expert systems in the battle against COVID-19 disease. This study explores different types of AI applications that support humans to diagnose and suppress the extensive impacts of the outbreak. A combination of expert system and fuzzy logic is studied and suggested regarding diagnosing early symptoms of this virus and fuzzy expert system and its usage in different similar situations is discussed. Besides, this study presents a suggestion for COVID-19 diagnosis to facilitate future studies about this disease.

\section{ARTIFICIAL INTELLIGENCE AND COVID-19}

The expert system can be used to detect the most common symptoms of COVID -19 , which are fever, tiredness, and dry cough. Having aches and pains, nasal congestion, runny nose, sore throat, or diarrhoea are some other symptoms that can consider in the expert system. The symptoms begin slowly; around 1 out of 6 patients becomes seriously ill with the serious breathing issue. Older people, and those with high blood pressure, heart problems, or diabetes would need more medical attention ${ }^{4}$. The expert systems can be used for early diagnosis which consists of the knowledge base, interference engine, and user interface.,

The first well-known expert system is MYCIN developed in 1972 at Stanford University in California ${ }^{7}$. Even at the period, this expert system could do wonders such as requesting further information concerning the patient and suggesting additional laboratory tests to arrive at a probable diagnosis, after which it would recommend a course of treatment. If requested to MYCIN, it would also explain the reason behind the result of the diagnosis. Similarly, some features of MYCIN can be implemented in the proposed expert system. For example, if a patient is not satisfied with the diagnosis of an expert system, the expert system could suggest the appropriate test that should be done, the kind of treatments they should follow and the medicines they should take.
Another expert system by the name of Oncocin is a rulebased medical expert system for oncology protocol management developed at Stanford University. ${ }^{8}$ This expert system was designed to assist physicians with the treatment of cancer patients receiving chemotherapy. Similarly, some of the proposed systems could have this kind of feature. It should be considered that some COVID-19 patients might have very severe symptoms that might worsen without the proper treatment. The expert system can help to store the required treatment that was used to treat a patient that has a severe COVID -19 symptoms and might assist the physicians in the future in treating the COVID - 19 patients to prevent the symptoms from worsening.

\section{EXPERT SYSTEM}

The expert system helps doctors and people to detect and diagnosis the symptoms that may face in real-time, without losing a second. Considering the main components of the expert system, the knowledge base of the expert system can be obtained from the doctors, nurses, and researchers that primarily handling this virus cases. This kind of expert system not only detects the virus earlier but also reduces the workload of the frontline workers in the medical field to diagnose the potential patients. With a faster detection of the virus, there will be lesser deaths among the COVID -19 patients, and more patients would recover at a rapid rate since detecting the virus earlier would increase the recovery rate among the patients. Therefore, the doctors, nurses, and researchers who are working with COVID - 19 cases can be considered as domain experts in this research to obtain information about the COVID -19 virus. Once the knowledge is obtained, the expert system will convert and keep the information in its knowledge-based expert systems. All the symptoms including the common or not so common symptoms are saved in the knowledge-based. Then the interference engine should be able to combine both symptoms to determine whether the patient is suffering from COVID - 19 or the common flu. To enhance user interaction with the system, graphical user interfaces should be employed as well into the system. This would make it easier for anyone to use the system and not requiring any medical officer to help the public in assisting them to operate the system.

\section{Fuzzy Expert System}

In disease diagnostic process, the fuzzy expert system uses the combination of expert systems and fuzzy logic to diagnose early symptoms. ${ }^{9}$ For example, the fuzzy expert system that is used in diagnosing early symptoms of bird flu is a hybrid intelligent system to solve decision making and interference problems in the presence of uncertainty. This approach includes 4 main processes which include the knowledge base, fuzzification, fuzzy interference, and defuzzification. 
The primary source of information on the knowledge base would be the doctors. In the fuzzification process, the input variable that is entered by the user will be transformed into a linguistic variable. The fuzzy expert system and its components. The user needs to enter the degree of his/her fever from a scale of 1 to 100 , this will become the input. A piecewise linear function is used since this is the simplest type of membership function (MFs), yet powerful enough to classify various linguistic values. Under the fuzzy interference process, the truth value for the premise of each rule is computed and applied to the concluding section of each rule. This results in one fuzzy subset to be assigned to each output variable for each rule. And the last process would be defuzzification, which is used to convert the fuzzy output set to a crisp number. The centre of area (COA) techniques which is a central value in the universe of discourse and that due to its complexity it may lead to a rather slow inference cycle. Once all symptoms have been key in based on percentages of probability on each symptom, then the system calculates the percentage that the user has been infected.

Another expert system used for the early diagnosis of eye diseases infecting the Malaysian population consists of the knowledge base, interference engine, and user interface. ${ }^{5}$ The knowledge base is acquired from sources such as relevant medical documents, nurses, patients, and ophthalmologists. The interference engine allows between the user and the system. The knowledge acquisition goes through 8 different levels which include literature study, questionnaire, pilot survey, and improved questionnaire interview with patients, interview with ophthalmologists, extracting the knowledge from the 6 stages, and finally inserting into the knowledge base. The expert system used for early diagnosis of human disease is rule-based and makes inferences with symbols, which require translation of a diseases specific knowledge in the standard symbolic form. ${ }^{10}$ Firstly, the medical background of disease is recorded from a personal interview with doctors and patients, then a set of rules are created and the interference engine will analyze the information based on the rules created. With the expert system, the user can interact with a computer to solve a certain problem. This can occur because the expert system can store heuristic knowledge.

\section{DISCUSSION AND RECOMMENDATION}

Based on the literature review, there are a couple of ways in diagnosing COVID - 19 patients who have symptoms at an early stage. As it has been discussed, the fuzzy expert system does not make decisions based on black or white, it often involves grey areas and the term may be as in diagnosing a potential COVID-19 patients, there is no such a very specific symptom like dengue where the main symptom is high fever. Based on the previous COVID-19 patients, symptoms vary from one patient to another such as fever, flu, diarrhoea, sore throat, and many other symptoms. Thus, with this approach, the system is also able to make decisions even in a complex situation. As discussed earlier, the fuzzy expert system implements the fuzzification process where the membership functions defined on the input variable apply to their actual values. This helps to determine the degree of truth for each rule. Each rule represents a symptom. Every disease has a specific degree of symptoms at an early stage that even domain experts such as doctors may not know at that early stage. For example, all H1N1 virus (swine flu) patients also might have flu and fever symptoms, but there are very fewer people who suffer from fever and the temperature of the person usually does not go up so high. ${ }^{11}$ Even dengue patients have fever symptoms but patients who suffer from dengue will have a high and sudden fever. ${ }^{12}$ This clearly shows that even there are some symptoms similar between dengue fever and the H1N1 virus, but the degree of the symptom differs. Similarly, there might be a certain degree of symptoms that can detect COVID-19 patients at an early stage, and fuzzification can make the diagnostic process easier.

The knowledge acquisition on the expert system is one of the best ways to acquire information; as there are 8 different levels to fill up its knowledge base. ${ }^{5}$ Dealing with COVID-19, the knowledge base should have a thorough amount of knowledge to able the system to diagnose the patient at the early stage of the infection. Thus, when the knowledge base in the proposed expert system can be acquired from various sources such as domain expert, patients (including the recovered ones), questionnaire, surveys and nurses then, the expert system will have a wider and broader range of knowledge. This will help the decision-making process faster. The proposed system can also provide suggestions on how to reduce the spread of the virus as well as to speed up the recovery in COVID-19 patients.

The heuristic approach used in the expert system diagnosing the human disease is an excellent method in doing early diagnosing on COVID-19 patients. ${ }^{10}$ No matter how deep or wide the knowledge base is created, some crucial information about some symptoms of the disease might be left out. Furthermore, for the special cases of the patients with a history of medical problems like high blood pressure, heart and lung problems, diabetes, or cancer, the domain expert might not know the exact early symptoms of the COVID-19. The mutation of the virus also can make the virus more aggressive ${ }^{13}$; new various kinds of symptoms can occur and the domain expert may not be able to identify the new symptoms. Thus, with a heuristic approach, the expert system will be able to learn from their own experience from the COVID-19 patients and with that experience, the expert system will be able to identify early symptoms on patients who are already suffering from certain medical problems and patients even if they get infected by the mutated version of the virus. ${ }^{14}$ 


\section{CONCLUSIONS}

The use of the fuzzy expert system would help to diagnose COVID -19 virus at a much early stage. It will give more accurate results compared to the doctor's diagnosis. The benefits will be gained by both doctors and patients as the doctors will be able to save their time in diagnosing patients and focus more on treating COVID-19 patients and patients will be able to diagnose themselves and get the appropriate treatment as soon as possible. Patients can use the system on their own too which can save their time and money to refer to medical centres. Only when the system states that the patient is suffering from COVID -19, then the patient can seek medical attention from the nearest hospital. This expert system can be placed anywhere such as bus stations, train stations, airports, grocery shops, and even installed in personal devices. The feature of virtual voice assistant can also be considered for using the expert system more efficiently.

\section{REFERENCES}

1. The Star Online. 2020. Covid-19: Patient 26'S Higher Infection Rate Could Be Due To Virus Mutation. [online] Available at: $<$ https:/www.thestar.com.my/news/nation/2020/04/29/covid19-patient-26039s-higher-infection-rate-could-be-due-to-virusmutation $>$ [Accessed 29 April 2020].

2. Who.int. 2020. Coronavirus. [online] Available at: $<$ https:// www.who.int/health-topics/coronavirus\#tab=tab_1> [Accessed 23 April 2020].

3. Npr.org. 2020. NPR Choice Page. [online] Available at: $<$ https:/www.npr.org/sections/health-shots/2020/03/28/822869504/ why-it-takes-so-long-to-get-most-covid-19-test-results $>$ [Accessed 23 April 2020]
4. Detail Question and Answers on COVID-19 for Public, 2020. https://www.mohfw.gov.in/pdf/FAQ.pdf.

5. Ibrahim F, Basheer AJ, Jaais F, Taib M. Expert System for Early Diagnosis of Eye Diseases Infecting the Malaysian Population. IEEE Catalogue No. 01 CH37239, 2020; 3(2): 123

6. Mozaharul DM, Rahman Md, Habib Md, Ahmed F. Detection of the Onset of Diabetes Mellitus by Bayesian Classifier Based Medical Expert System. Transact Machine Learning Artifi Intel 2016;4:1-8.

7. Copeland BJ. Mycin, artificial intelligence program. 2016. https://www.britannica.com/technology/MYCIN

8. Wiederhold GE, Shortliffe L. Fagan I, Perreault L, Medical Informatics: Computer Applications in Health Care and Biomedicine. 2nd Edn., Springer, New York, ISBN-10: 0387984720, 2016; 854.

9. Razak TRB, Othman MB, Abu Bakar MN, Ahmad KA, Mansor AR. Fuzzy Expert System for Early Symptoms Detection of Bird Flu Disease. IEEE Symposium on Humanities Sci Engg Re. 2013.

10. Sahu PD, Mandal I. An Expert System For Diagnosis Of Human Diseases. 2012 [online] Available at: <https://www.researchgate.net/publication/43763822> [Accessed 29 April 2020].

11. Mayo Clinic. Swine Flu (H1N1 Flu)-Symptoms And Causes. [online] Available at:2020. $<$ https://www.mayoclinic.org/diseases-conditions/swine-flu/symptoms-causes/syc-20378103> [Accessed 28 April 2020].

12. WebMD. 2020. Dengue Fever. [online] Available at: $<$ https:// www.webmd.com/a-to-z-guides/dengue-fever-reference\#1> [Accessed 29 April 2020].

13. The Star Online. 2020. Covid-19: Most Deaths Due To Late Treatment, Says Health DG. [online] Available at: <https:// www.thestar.com.my/news/nation/2020/03/24/covid-19-mostdeaths-due-to-late-treatment-says-health-dg $>$ [Accessed 22 April 2020].

14. Scialert.net. 2020. An Expert System For Endocrine Diagnosis And Treatments Using JESS - Scialert Responsive Version. [online] Available at: $<$ https://scialert.net/fulltextmobile/?doi $=$ j ai.2010.239.251> [Accessed 28 April 2020]. 